\title{
Infecciones del sistema nervioso central asociadas a dispositivos de derivación de LCR en niños, en un centro neuro-quirúrgico de referencia nacional
}

\section{Central nervous system infections associated with CSF shunt devices in children, at a national reference hospital}

José Pablo Fernández V.1,2, Natalia Acosta G. ${ }^{1,2}$, Andres Goycoolea ${ }^{1,3}$ y Osvaldo Koller C. ${ }^{1,3}$

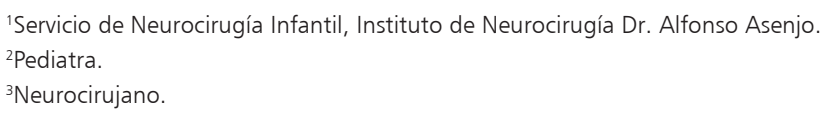

Financiamiento: no hubo.

Los autores declaran no tener conflicto de interés alguno.

Recibido: 6 de enero de 2021 / Aceptado: 19 de marzo de 2021

\section{Resumen}

Introducción: Las infecciones asociadas a dispositivos de derivación de LCR son una complicación frecuente en su utilización. Lo más habitual es la presencia de cocáceas grampositivas, como Staphylococcus coagulasa negativa (50\% en algunas series) y Staphylococcus aureus. Esta complicación agrega morbimortalidad al paciente neuroquirúrgico, aumentando la estadía hospitalaria y los costos de tratamiento. Objetivo: Conocer la incidencia de infecciones asociadas a dispositivos de derivación de LCR en un centro de referencia nacional. Metodología: Estudio descriptivo, retrospectivo. Se recolectó la información de los pacientes pediátricos (bajo 18 años) entre 2018 y 2019. Se realizó un análisis estadístico descriptivo e inferencial utilizando el lenguaje estadístico R 3.4.0 y RStudio 1.3.9. Se calculó la incidencia acumulada para cada procedimiento, evaluando si existe diferencias significativas entre ellas. Estudio aprobado por el Comité de Ética Pediátrico del SSMO. Resultados: En el período estudiado se realizaron 175 cirugías. Encontramos 19 casos de ventriculitis asociada a derivativa ventriculo-peritoneal y 7 casos en derivativa ventricular-externa. Los agentes más frecuentes fueron las cocáceas grampositivas. No se logró identificar factores de riesgo significativos.

Palabras clave: ventriculitis; derivación ventrículo-peritoneal; disfunción valvular.

\section{Abstract}

Background: Infections associated with CSF shunt devices are a frequent complication in their use. The most common is the presence of gram positive coccaceae, such as coagulase negative Staphylococcus (50\% in some series) and Staphylococcus aureus. This complication adds morbidity and mortality to the neurosurgical patient, increasing hospital stay and treatment costs. Aim: To determine the incidence of infections associated with CSF shunt devices in a national referral center. Methods: Retrospective, descriptive study. Information was collected on pediatric patients between 2018 and 2019. A descriptive and inferential statistical analysis was performed using the statistical language R 3.4.0 and RStudio 1.3.9. The cumulative incidence for each procedure was calculated, evaluating whether there were significant differences between them. This study was approved by the Pediatric Ethics Committee of the SSMO. Results: In the period studied, 175 surgeries were performed. We found 19 cases of ventriculitis associated with ventriculoperitoneal derivative and 7 cases in ventricular-external derivative. The most frequent agents were grampositive coccaceae. It was not possible to identify significant risk factors.

Keywords: cerebral ventriculitis; ventriculo-peritoneal shunt; shunt malfunction. 


\section{Introducción}

E s habitual en Neurocirugía la utilización de dispositivos para drenar líquido cefalorraquídeo (LCR) de la cavidad ventricular. Estos pueden usarse de forma transitoria para drenar LCR, sangre, pus o para monitorizar la presión intracraneal (drenaje ventricular externo DVE). También pueden ser utilizados de forma prolongada como manejo de la hidrocefalia, drenando LCR a la cavidad peritoneal, a la pleura o aurícula derecha, entre otros sitios (drenaje ventrículo peritoneal -DVP-o según donde drene $)^{1}$. Estos son susceptibles de colonizar y causar una infección del ventrículo y el parénquima cerebral.

La frecuencia de estas complicaciones es variable en la literatura médica y depende de los criterios a utilizar y el tipo de dispositivo estudiado. En el caso de las derivaciones internas, la frecuencia de infección se estima de 4 a $17 \%{ }^{2,3}$ y de $8 \%$ para los drenajes ventriculares externos ${ }^{4}$.

\section{Fisiopatología}

El factor más relevante en la infección de las DVP es la colonización del catéter durante la instalación en pabellón, por lo que la mayoría de las infecciones se manifiesta dentro de un mes desde la instalación. Un estudio prospectivo identificó agujeros en los guantes y la manipulación, con las manos, del dispositivo como un posible factor de riesgo, además de las fístulas de LCR y la condición de prematuridad ${ }^{5}$.

Los DVE actúan como una puerta de entrada directa al ventrículo, por lo que el tiempo de permanencia ${ }^{6}$ y la manipulación excesiva ${ }^{7}$ han mostrado aumentar el riesgo de infección. Además, la filtración de $\mathrm{LCR}^{8}$ y la gravedad del paciente son factores de riesgo'.

En un estudio nacional se encontraron como factores de riesgo de infección el antecedente de un anterior episodio de disfunción e infección de la válvula, otra infección concomitante y que el procedimiento hubiera sido realizado por un neurocirujano no formado en la disciplina pediátrica ${ }^{10}$.

\section{Microbiología}

Las cocáceas grampositivas corresponden a la causa más frecuente de infección, siendo las más habituales los agentes que colonizan la piel, como Staphylococcus coagulasa negativa $(50 \%$ en algunas series) y Staphylococcus aureus. En DVE se pueden encontrar bacilos gramnegativos, como Pseudomonas aeruginosa y Klebsiella pneumoniae. En menor proporción se encuentran Propionibacterium acnes, hongos como Candida spp y otros agentes como etiología $a^{11-13}$.

\section{Diagnóstico}

Se debe sospechar una infección ventricular en un paciente portador de DVP que presenta fiebre, habitualmente asociada a síntomas de disfunción valvular, como son la cefalea, náuseas o compromiso de conciencia ${ }^{14}$. Además, pueden presentar déficit neurológico focal, rigidez de nuca, convulsiones y fotofobia. En un paciente con DVP, la signología abdominal debe hacer sospechar una infección del dispositivo. La demostración de bacteriemia sin otra causa, en un paciente con derivativa ventrículo atrial, también debe hacer pensar en ventriculitis ${ }^{15}$.

Los exámenes citoquímicos realizados de rutina al LCR tienen moderada utilidad, ya que algunas alteraciones pueden ser secundarias a la causa que motivó la derivación (proteínas elevadas, hemorragia). A su vez, un LCR sin alteraciones, no descarta una infección ventricular $^{16,17}$. El lactato bajo en LCR, tiene un buen valor predictor negativo $^{18,19}$.

El cultivo de LCR del drenaje o del reservorio de la DVP es el estudio más importante para confirmar el diagnóstico, a pesar de que su rendimiento es de aproximadamente $50 \%$, según estudios internacionales ${ }^{20}$. La utilización de reacción de polimerasa en cadena (RPC) permite pesquisar aproximadamente $50 \%$ de los casos en que el cultivo fue negativo ${ }^{21}$. Los hemocultivos tienen un rendimiento menor a $20 \%$, excepto en las derivaciones al atrio cardíaco, donde la bacteriemia es de regla.

El diagnóstico se debe sospechar en un paciente portador de una DVP o un drenaje externo, con manifestaciones clínicas compatibles, asociada a pleocitosis, glucorraquia baja y proteínas elevadas en el LCR. En caso de dudas se puede repetir el análisis del LCR, apoyando el diagnóstico un aumento de las células o una baja de la glucosa. Un cultivo positivo con un agente compatible confirma el diagnóstico 22

\section{Tratamiento}

La Infectious Diseases Society of America (IDSA) recomienda iniciar tratamiento antimicrobiano de forma empírica, si la expresión clínica y la pleocitosis del LCR hacen sospechar una infección ventricular. Se deben utilizar antimicrobianos asociados, con cobertura para los agentes causales más frecuentes y con buena penetración en el SNC. Se recomienda iniciar vancomicina para alcanzar concentraciones plasmáticas de $15-20 \mu \mathrm{g} / \mathrm{dL}$ y un antimicrobiano con cobertura para bacilos gramnegativos, como cefalosporinas con actividad anti-pseudomonas, meropenem $\mathrm{u}$ otros, $\mathrm{etc}^{23}$. Debe diseñarse esta terapia empírica inicial según la epidemiología institucional y ajustarse una vez que se conozca la etiología y susceptibilidad bacteriana in vitro.

El dispositivo infectado debe ser retirado, manteniendo un drenaje ventricular externo hasta negativizar los cultivos de $\mathrm{LCR}^{24}$. La duración del tratamiento depende de la inflamación del líquido y el agente identificado. Con un LCR sin pleocitosis y cultivos positivos a Staphylococcus coagulasa negativa, la IDSA recomienda 10 días 
de antibioterapia. Para S. aureus, deben completarse al menos 14 días. Con cultivos positivos persistentes, debe extenderse el tratamiento por al menos dos semanas después de obtener su negativización ${ }^{25}$. La reinstalación de la válvula se podría realizar luego de $48 \mathrm{~h}$ de tratamiento (Staphylococcus coagulasa negativa sin pleocitosis en el LCR) o esperar 7-10 días en caso de pleocitosis del LCR, (S. aureus o bacilos gramnegativos $)^{26,27}$.

\section{Objetivo primario}

Conocer la epidemiología local de infecciones ventriculares asociadas a derivativa ventricular y DVE en un centro de referencia nacional.

\section{Objetivos secundarios}

- Describir la población afectada por infecciones ventriculares asociadas a derivativas ventriculares y DVE.

- Relacionar el tipo de cirugía con la frecuencia de infecciones.

- Recopilar los cultivos positivos, mostrando los agentes más frecuentes y su patrón de resistencia antimicrobiana.

\section{Pacientes y Métodos}

Estudio descriptivo, retrospectivo. Se obtuvo la información sobre infecciones ventriculares asociadas a dispositivos de drenaje de LCR, de pacientes ingresados a los servicios de Neurocirugía Pediátrica y Unidad de Cuidados Intensivos Pediátricos del Instituto de Neurocirugía Dr. Alfonso Asenjo durante los años 2018 y 2019.

Del Departamento de Estadísticas hospitalarias se recolectó la información respecto a los pacientes ingresados y egresados con los diagnósticos de ventriculitis y meningitis. Por ser éste un centro quirúrgico, no ingresaron pacientes con infecciones del SNC no relacionadas a dispositivos.

Se rescataron del Laboratorio de Microbiología del Hospital Del Salvador (institución vecina con convenio para el laboratorio de microbiología) los cultivos de LCR positivos de pacientes ingresados, registrando los agentes identificados y su patrón de resistencia antimicrobiana.

Para evitar pérdidas de datos, se solicitó de la Unidad de Farmacia el listado de pacientes que recibieron tratamiento antimicrobiano según el esquema habitual de uso en nuestra institución, buscando casos que pudieran no haber sido registrados inicialmente.

La información sobre las cirugías fue obtenida de los registros del servicio.

\section{Definiciones}

- $1^{\circ}$ DVP: a la instalación de este dispositivo a un paciente por primera vez, independiente de la causa.
- Revisión de DVP: procedimiento de exposición, revisión manual, y en ocasiones reemplazo, de sus componentes para tratar la obstrucción: catéter proximal, válvula o catéter distal.

- Externalización DVP: exteriorización del catéter proximal o distal para solucionar la obstrucción.

- Instalación DVE: procedimiento en el cual se instala un catéter desde el ventrículo al exterior, retirando el sistema de drenaje previo (si existe).

Se excluyeron pacientes cuyas DVP no hubieran sido instaladas en nuestro centro. Además, se excluyeron del análisis los casos donde la DVP se infectó de forma secundaria (ejemplo: proceso abdominal) y los pacientes con diagnóstico de absceso cerebral.

Se registraron los datos obtenidos en una base de datos Google Spreadsheet creada para tal efecto. Esta fue protegida con contraseña y sólo fue accesible para los autores.

La identificación microbiana se realizó con un equipo Vitek MS (Biomerieux) y el estudio de antibiograma en Vitek 2 compact (Biomerieux).

Se realizó un análisis estadístico descriptivo e inferencial, utilizando el lenguaje estadístico R $3.4 .0^{28}$ y RStudio 1.3.929. Se calculó la incidencia acumulada para cada procedimiento y según la causa de instalación, evaluando si existieron diferencias significativas entre ellas.

\section{Ética}

Estudio aprobado por el Comité de Ética Pediátrico del SSMO. Dado el carácter retrospectivo y el no presentar riesgo para los pacientes, se autorizó a prescindir del Consentimiento Informado, cuando no fuera posible obtenerlo luego de tres intentos frustros de contactar a los apoderados de los pacientes.

\section{Resultados}

En el período entre enero de 2018 y diciembre de 2019 se realizaron 175 procedimientos con DVP y 91 con DVE (total: 266), en 134 pacientes. Lo más frecuente fueron los recambios de DVP y la instalación de un nuevo DVE (Figura 1). La media de edad fue 73,2 meses (6,1 años) (mínimo 1 mes, máximo 16 años), con 49,6\% de mujeres. La indicación más frecuente para instalación de DVP y DVE fue hidrocefalia secundaria a tumor e hidrocefalia congénita.

En el grupo de las DVP, se presentaron 19 casos de infección ventricular en el período estudiado (10,8\% del total de DVP). La frecuencia de infección según la causa de hidrocefalia (y por la cual se instaló la DVP) se detalla en la Tabla 1. No hubo diferencias significativas entre ellas. En los pacientes en quienes se instaló por primera 


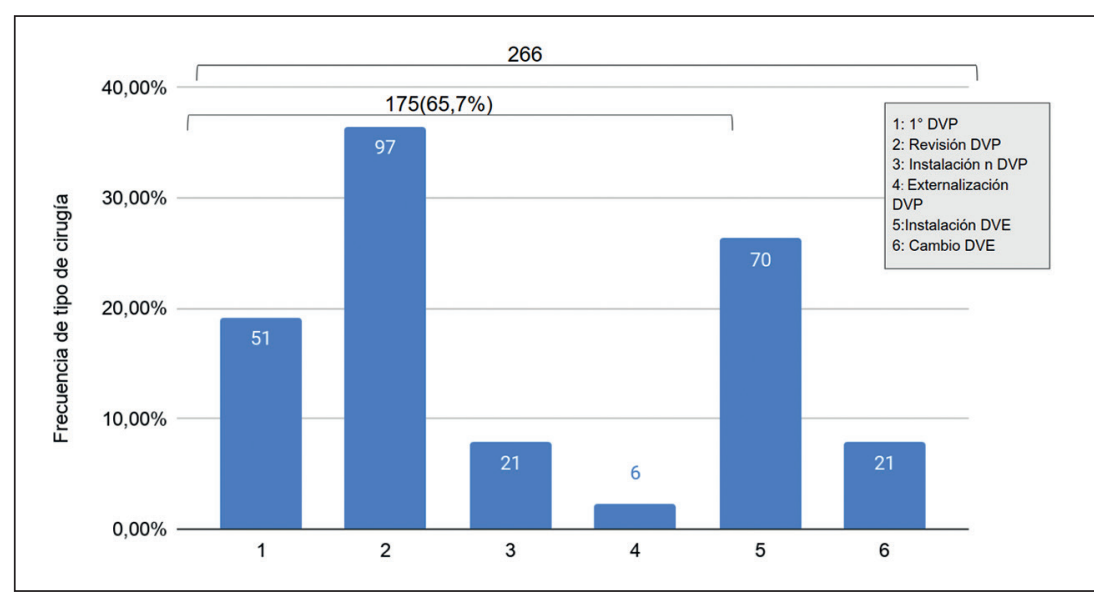

Figura 1. Tipo de cirugía efectuada en derivaciones de LCR en niños (n: 266).

Tabla 1. Cirugía derivativa ventrículo-peritoneal de LCR en niños. Tasa de infección según causa de la hidrocefalia

\begin{tabular}{lccc}
\hline Etiología HCF & $\mathbf{n}$ de casos & Ventriculitis & Tasa de incidencia \\
\hline $2^{\circ}$ a tumor & 53 & 4 & $7,55 \%$ \\
Hidrocefalia congénita & 45 & 7 & $15,56 \%$ \\
Mielomeningocele & 27 & 4 & $14.81 \%$ \\
$2^{\circ}$ a infección & 12 & 1 & $8,33 \%$ \\
Quiste aracnoidal & 8 & 2 & $25,0 \%$ \\
\hline Idiopática & 7 & 0 & 0 \\
$2^{\circ}$ a hemorragia & 3 & 0 & 0 \\
$2^{\circ}$ a hemorragia intraventricular & 1 & 1 & $5,56 \%$ \\
$2^{\circ}$ a TEC & 1 & 0 & 0 \\
Otros & 1 & 0 & 0 \\
\hline Total & 175 & 19 & $10,86 \%$ \\
\hline
\end{tabular}

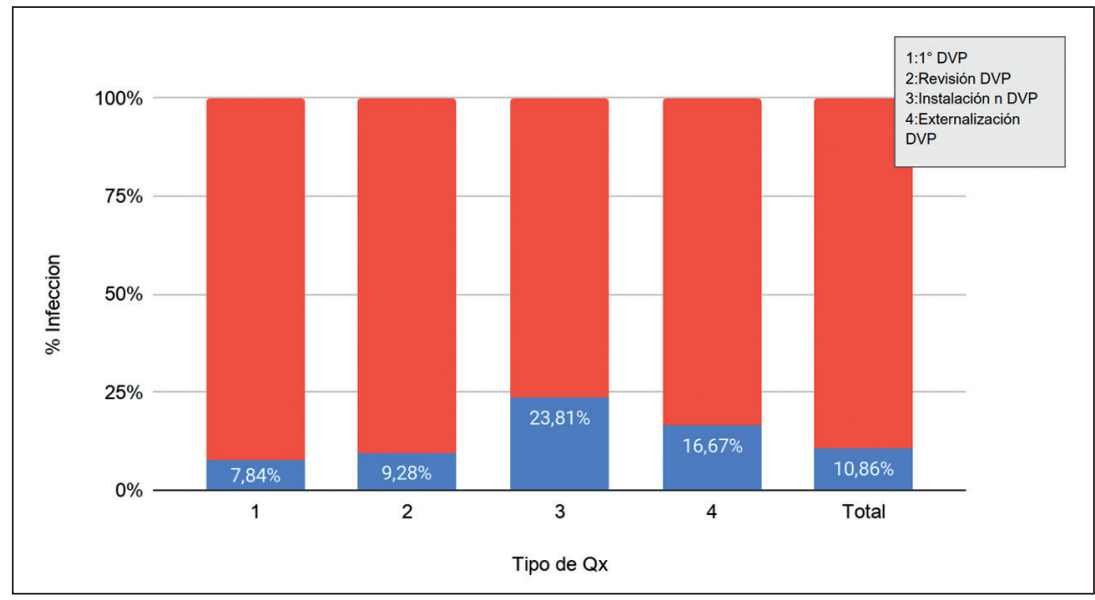

Figura 2. Porcentaje de infección según tipo de cirugía en derivaciones de LCR en niños. (n: 175). vez una DVP, la incidencia de infección fue de 7,8\%, aumentando hasta $23,8 \%$ en las instalaciones a repetición, siendo esta diferencia no significativa $(\mathrm{p}=0,14)$ (Figura 2). Tampoco hubo diferencias significativas en las tasas de ventriculitis entre hombres y mujeres ni en menores y mayores de dos años de edad.

En las DVE, se pesquisaron siete casos de ventriculitis, (6,59\% de los casos) siendo por igual si la causa del DVE fue por hidrocefalia o por hemorragia.

En las ventriculitis secundarias a DVP, los agentes más frecuentemente encontrados fueron cocáceas grampositivas, con una positividad en los cultivos de $84 \%$. Los microorganismos encontrados y sus patrones de susceptibilidad in vitro se detallan en la Tabla 2. En las infecciones de DVE, sólo se obtuvieron tres aislamientos positivos en los siete casos. No hubo aislamiento de $P$. acnes ni $P$. aeruginosa en el período estudiado.

Los 19 pacientes con ventriculitis por DVP comenzaron tratamiento según el esquema institucional: ceftriaxona y vancomicina endovenosas en dosis meníngeas. Por persistencia de cultivos positivos al $7^{\circ}$ día, se inició tratamiento intratecal con vancomicina en cinco pacientes, negativizando en todos los casos, el cultivo de LCR tras 7 días de tratamiento. Ninguno de los pacientes con infección del DVE requirió tratamiento intratecal.

\section{Discusión}

Este estudio tuvo como objetivo realizar una primera descripción de las infecciones asociadas a dispositivos de derivación de LCR en un centro de referencia nacional. Dado el bajo número de casos, no encontramos asociación con los factores de riesgo descritos en la literatura médica, como es la edad. Tampoco hubo diferencia entre el sexo de los pacientes. La incidencia fue similar a la reportada por otros autores (hasta 17\%). A nuestro conocimiento, en otros artículos similares no se diferencia entre la instalación de la $1^{\circ} \mathrm{DVP}$ y las instalaciones a repetición.

Las diferencias encontradas en los casos de ventriculitis, según cada patología, pueden deberse a que en las que presentaron mayor proporción de casos de infección suelen requerir mayor número de cirugías y disfuncionar más frecuentemente. Aquello se refleja en que la instalación de DVP a repetición fue el único factor de riesgo de ventriculitis que identificamos, lo que es concordante con la literatura médica, a pesar de que un $\mathrm{n}$ bajo no nos permitió encontrar una significancia estadística.

Fueron pocos casos de infección en las derivativas externas, lo cual no nos permitió determinar factores 
de riesgo. Esto puede deberse a que existe experiencia en nuestro centro en el equipo médico, de enfermería y técnico, para el manejo de estos drenajes, logrando que su incidencia sea menor a la descrita en la literatura correspondiente.

El aislamiento microbiológico es concordante con lo publicado en otros estudios, con una mayor incidencia de cocáceas grampositivas. No hubo casos con aislamiento de $P$. acnes ni $P$. aeruginosa, a diferencia de lo descrito en otros artículos.

\section{Conclusión}

Este artículo busca ser el primer reporte de nuestro equipo de Neurocirugía Infantil sobre el estudio esta patología. Al ser el centro con mayor volumen de pacientes neuro-quirúrgicos pediátricos, nos permite aportar al conocimiento para la prevención, diagnóstico y manejo adecuado de las ventriculitis asociadas de DVP y DVE.

Agradecimientos: A Ricardo Morales, infectólogo de adultos, en la revisión de este artículo.

\begin{tabular}{|c|c|c|c|c|}
\hline Cultivo & Agente & Oxacilina & Rifampicina & $\begin{array}{l}\text { Vancomicina } \\
\text { CIM } \mu / \mathrm{mL}\end{array}$ \\
\hline 1 & S. epidermidis & $\mathrm{R}$ & $\mathrm{R}$ & $\mathrm{S}(\mathrm{CIM}=1)$ \\
\hline 2 & S. epidermidis & $R$ & S & $\mathrm{S}(\mathrm{CIM}<0,5)$ \\
\hline 3 & S. epidermidis & $\mathrm{R}$ & s & $\mathrm{S}(\mathrm{CIM}=1)$ \\
\hline 4 & S. coagulasa negativa & $S$ & S & $\mathrm{S}(\mathrm{CIM}=0,5)$ \\
\hline 5 & S. coagulasa negativa & $\mathrm{NI}$ & S & $\mathrm{NI}$ \\
\hline 6 & S. coagulasa negativa & $\mathrm{R}$ & R & $\mathrm{S}(\mathrm{CIM}=2)$ \\
\hline 7 & S. coagulasa negativa & $\mathrm{R}$ & S & $\mathrm{S}(\mathrm{CIM}=1)$ \\
\hline 8 & S. coagulasa negativa & $\mathrm{R}$ & S & $S(C I M=1)$ \\
\hline 9 & S. capitis & S & S & $\mathrm{S}(\mathrm{CIM}=1)$ \\
\hline 10 & S. aureus & S & s & $S(C I M<0,5)$ \\
\hline 11 & S. aureus & S & S & $\mathrm{S}(\mathrm{CIM}<0,5)$ \\
\hline 12 & S. aureus & S & S & $S(C I M<0,5)$ \\
\hline 13 & S. aureus & $\mathrm{S}$ & $S$ & $\mathrm{~S}(\mathrm{CIM}=1)$ \\
\hline 14 & E. faecalis & $\begin{array}{c}\mathrm{NI} \\
\text { Amikacina }\end{array}$ & $\begin{array}{c}\mathrm{NI} \\
\text { Ceftazidina }\end{array}$ & $\begin{array}{l}\mathrm{S}(\mathrm{CIM}=2) \\
\text { Meropenem }\end{array}$ \\
\hline 15 & A. baumannii & S & S & S \\
\hline 16 & A. baumannii & s & S & S \\
\hline 17 & Negativo & & & \\
\hline 18 & Negativo & & & \\
\hline 19 & Negativo & & & \\
\hline
\end{tabular}

\section{Referencias bibliográficas}

1.- Kahle K T, Kulkarni A V, Limbrick D D, Warf B C. Hydrocephalus in children. Lancet 2016; 387: 788-99. https://doi. org/10.1016/s0140-6736(15)60694-8.

2.- Van de Beek D, Drake J M, Tunkel A R. Nosocomial bacterial meningitis. N Engl J Med. 2010; 362: 146-54. https://doi. org/10.1056/NEJMra0804573.

3.- Vinchon M, Dhellemmes P. Cerebrospinal fluid shunt infection: risk factors and long-term follow-up. Childs Nerv Syst 2006; 22: 692-7. https://doi.org/10.1007/ s00381-005-0037-8.

4.- Lozier A P, Sciacca R R, Romagnoli M F, Connolly E S. Ventriculostomyrelated infections: a critical review of the literature. Neurosurgery 2008; 62 Suppl 2: 688-700. https://doi.org/10.1227/01. neu.0000316273.35833.7c

5.- Kulkarni A V, Drake J M, Lamberti-Pasculli M. Cerebrospinal fluid shunt infection: a prospective study of risk factors. J Neurosurg. 2001; 94: 195-201. https://doi.org/10.3171/ jns.2001.94.2.0195.

6.- Jamjoom A A B, Joannides A J, Poon M T C, Chari A, Zaben M, Abdulla M A H, et al.
Prospective, multicentre study of external ventricular drainage-related infections in the UK and Ireland. J Neurol Neurosurg Psychiatry 2018; 89: 120-6. https://doi.org/10.1136/jnnp2017-316415.

7.- Hoefnagel D, Dammers R, Ter Laak-Poort M P, Avezaat C J J. Risk factors for infections related to external ventricular drainage. Acta Neurochir (Wien) 2008; 150: 209-14; discussion 214. https://doi.org/10.1007/s00701007-1458-9.

8.- Park J, Choi Y-J, Ohk B, Chang H-H. Cerebrospinal fluid leak at percutaneous exit of ventricular catheter as a crucial risk factor for external ventricular drainage-related infection in adult neurosurgical patients. World Neurosurg. 2018; 109: e398-403. https://doi.org/10.1016/j. wneu.2017.09.190.

9.- Federico G, Tumbarello M, Spanu T, Rosell R, Iacoangeli M, Scerrati M, et al. Risk factors and prognostic indicators of bacterial meningitis in a cohort of 3580 post-neurosurgical patients. Scand J Infect Dis. 2001; 33: 533-7. doi: 10.1080/00365540110026557.

10.- Pena A A, Sandia Z R, Riveros P R, Salazar Z C, Herrera O R, Vergara F R.
Factores de riesgo de infección de derivativa ventrículo peritoneal en pacientes pediátricos del Hospital Carlos van Buren. Rev Chilena Infectol. 2012; 29: 38-43. https://doi. org/10.4067/s0716-10182012000100007.

11.- Simon T D, Kronman M P, Whitlock K B, Browd S R, Holubkov R, Kestle J R W, et al. Patient and treatment characteristics by infecting organism in cerebrospinal fluid shunt infection. J Pediatr Infect Dis Soc. 2019; 8 (3): 235-43. doi: 10.1093/jpids/piy035.

12.- Yakut N, Soysal A, Kepenekli Kadayifci E, Dalgic N, Y1lmaz Ciftdogan D, Karaaslan A, et al. Ventriculoperitoneal shunt infections and re-infections in children: a multicentre retrospective study. Br J Neurosurg. 2018; 32: 196-200. https://doi.org/10.1080/02688697.201 8.1467373.

13.- Ramanan M, Lipman J, Shorr A, Shankar A. A meta-analysis of ventriculostomy-associated cerebrospinal fluid infections. BMC Infect Dis. 2015; 15. https://doi.org/10.1186/s12879-0140712-z.

14.- Tunkel A R, Hasbun R, Bhimraj A, Byers K, Kaplan S L, Scheld W M, et al. 2017 Infectious Diseases Society of America's Clinical Practice Guidelines for HealthcareAssociated Ventriculitis and Meningitis. Clin 
Infect Dis. 2017; 64: e34-65. https://doi. org/10.1093/cid/ciw861.

15.- Schoenbaum S C, Gardner P, Shillito J. Infections of cerebrospinal fluid shunts: epidemiology, clinical manifestations, and therapy. J Infect Dis. 1975; 131: 543-52. doi: 10.1093/infdis/131.5.543.

16.- Conen A, Walti L N, Merlo A, Fluckiger U, Battegay M, Trampuz A. Characteristics and treatment outcome of cerebrospinal fluid shunt-associated infections in adults: a retrospective analysis over an 11-year period. Clin Infect Dis. 2008 July 1; 47 (1): 73-82. doi: 10.1086/588298.

17.- Schade R P, Schinkel J, Roelandse F W C, Geskus R B, Visser L G, Van Dijk J M $\mathrm{C}$, et al. Lack of value of routine analysis of cerebrospinal fluid for prediction and diagnosis of external drainage-related bacterial meningitis. J Neurosurg. 2006; 104: 101-8. https://doi.org/10.3171/jns.2006.104.1.101

18.- Huy N T, Thao N T H, Diep D T N, Kikuchi M, Zamora J, Hirayama K. Cerebrospinal fluid lactate concentration to distinguish bacterial from aseptic meningitis: a systemic review and meta-analysis. Crit Care Lond Engl. 2010; 14: R240. https://doi.org/10.1186/cc9395.

19.- Sakushima K, Hayashino Y, Kawaguchi T, Jackson J L, Fukuhara S. Diagnostic accuracy of cerebrospinal fluid lactate for differentiating bacterial meningitis from aseptic meningitis: a meta-analysis. J Infect. 2011; 62: 255-62. https://doi.org/10.1016/j.jinf.2011.02.010.

20.- Srihawan C, Castelblanco R L, Salazar L, Wootton S H, Aguilera E, Ostrosky-Zeichner L, et al. Clinical characteristics and predictors of adverse outcome in adult and pediatric patients with healthcare-associated ventriculitis and meningitis. Open Forum Infect Dis. 2016; 3: ofw077. https://doi.org/10.1093/ofid/ofw077.

21.- Banks J T, Bharara S, Tubbs R S, Wolff C L, Gillespie G Y, Markert J M, et al. Polymerase chain reaction for the rapid detection of cerebrospinal fluid shunt or ventriculostomy infections. Neurosurgery 2005; 57: 123743; discussion 1237-1243. doi: 10.1227/01. neu.0000186038.98817.72

22.- Stenehjem E, Armstrong W S. Central nervous system device infections. Infect Dis Clin North Am. 2012; 26: 89-110. https://doi. org/10.1016/j.idc.2011.09.006.

23.- Seidelman J, Lewis S S. Neurosurgical devicerelated infections. Infect Dis Clin North Am 2018; 32: 861-76. https://doi.org/10.1016/j. idc.2018.06.006.

24.- Walters B C, Hoffman H J, Hendrick E B, Humphreys R P. Cerebrospinal fluid shunt infection. Influences on initial management and subsequent outcome. J Neurosurg 1984; 60: 1014-21. https://doi.org/10.3171/ jns.1984.60.5.1014.

25.- Kestle J R W, Garton H J L, Whitehead W E, Drake J M, Kulkarni A V, Cochrane DD, et al. Management of shunt infections: a multicenter pilot study. J Neurosurg. 2006; 105: 177-81. https://doi.org/10.3171/ped.2006.105.3.177.

26.- Cooke R P D, Pettorini B, Mallucci C. Prevention and management of neurosurgical shunt infections. J Hosp Infect. 2017; 95: 1612. https://doi.org/10.1016/j.jhin.2016.09.019.

27.- Arnell K, Cesarini K, Lagerqvist-Widh A, Wester T, Sjölin J. Cerebrospinal fluid shunt infections in children over a 13-year period: anaerobic cultures and comparison of clinical signs of infection with Propionibacterium acnes and with other bacteria. J Neurosurg Pediatr. 2008; 1: 366-72. https://doi. org/10.3171/PED/2008/1/5/366.

28.- R Core Team. R: A language and environment for statistical computing. Vienna, Austria: R Foundation for Statistical Computing; 2013. https://www.gbif.org/es/tool/81287/ra-language-and-environment-for-statisticalcomputing

29.- RStudio Team. RStudio: Integrated Development Environment for R. Boston, MA: RStudio, PBC.; 2020. http://www.rstudio.com/. 